\title{
Nonlinear Observer for the Control of Bi-Tethered Multi Aerial Robots
}

\author{
Marco Tognon ${ }^{1,2}$ and Antonio Franchi ${ }^{1,2}$
}

\begin{abstract}
We consider the problem of state-observation and control for a bi-tethered aerial system composed by a physical chain of two underactuated aerial robots, also called UAVs. The controlled outputs are the Cartesian position of the last robot and the internal forces along the links. We aim at a minimal use of sensors in order to retrieve the full state. For this goal we propose an output transformation method whose applicability implies the system observability. When this is the case we prove that it is possible to design a nonlinear state estimator based on the high gain- and Luenberger- observers that is able to retrieve the state from any dynamic condition. We also demonstrate how this estimator can be employed with a nonlinear controller for the Cartesian position and the link stresses while ensuring the stability in closed-loop. We show the validity of the method for sensorial configurations composed only by two accelerometers (no gyros) and just two encoders, or two accelerometers (no gyros) and just two inclinometers. A realistic simulative validation concludes the paper.
\end{abstract}

\section{INTRODUCTION}

The enormous popularity that aerial robots, commonly called UAVs (Unmanned Aerial Vehicles), are seeing in these days among the scientific community, but not only, is due to their versatility and applicability in a vast range of fields, such as search and rescue, surveillance, patrolling, agriculture, civil monitoring and so on.

Although their use as remote and unmanned sensors is dominant, they still present some limitations, as, e.g., short battery life and little payload, that impede their applicability in tasks requiring very long or even constant gathering of measurements. To solve this problem many recent works propose the tether solution. The use of a cable connecting the aerial vehicle to a fixed or mobile ground station can provide virtually endless energy and an high-bandwidth communication link to the robot, with applications in, e.g., monitoring [1] communication relay [2] and safe landing [3].

For tethered flight systems, works as [4] already proposed a controller to stabilize the elevation on a desired value using only onboard sensors. In [5] the positivity of the tension is also guaranteed during the transient. Finally, in [6] one can find a dynamic controller for both the link stress and elevation using a nonlinear observer based on inertial-only measurements. However, since the vehicle is constrained by the link to fly around the fixed point, the working space (a simple circle) can result very limited for such applications.

One solution is given by adding a second vehicle and a second link to the previous system. Indeed, in [7] is presented a system composed by two aerial robots connected to each others and to a fixed point by two links, forming an

\footnotetext{
${ }^{1}$ LAAS-CNRS, 7 Avenue du Colonel Roche, F-31400 Toulouse, France. mtognonelaas.fr, antonio.franchi@laas.fr

${ }^{2}$ Univ de Toulouse, LAAS, F-31400 Toulouse, France

This work has been partially funded by the European Union's Horizon 2020 research and innovation programme under grant agreement No 644271 AEROARMS.
}

actuated chain. The similarity with a planar two links robot immediately shows the advantages in terms of workspace, i.e., the second aerial robot in the chain (similarly to an end effector) can reach any point in the two dimensional working area, limited only by the full length of the system itself.

Using the controller proposed in [7] it is then possible to achieve the tracking of any desired Cartesian trajectory of the position of the second aerial robot while exactly controlling the stresses on the two links. This makes feasible its use in applications in, e.g., search end rescue, that were limited or impossible to accomplish with the single aerial robot case.

Though, in order to compute the control action in [7], the knowledge of the full-state is needed. In this work we study the observability of the full-state using a set of minimal sensors and show the design of an observer that can estimate the full-state from any dynamic condition using those sensors. This allows to close the control loop without the need of full-state measurements, thus decreasing the cost of the solution in terms of sensorial equipment.

More specifically, we propose here a method to transform the measurements coming from two onboard accelerometers and two encoders connected to the links into direct measures of a portion of the state. We then prove that the applicability of this method gives a sufficient condition for the observability of the system. In the case that the transformation is applicable, we propose a non linear observer that can work in closed loop with the controller presented in [7], while still preserving the stability of the global system. Finally we show how the same methodology can be easily employed for two other sensors configurations.

The paper is organized as follows. In Sec. II we recall the dynamic model of the system and the controller presented in [7]. The considered sensor configurations are presented as well. Then, in Sec. III we derive in details the nonlinear observer for a specific case, analyzing at the end the applicability of the proposed method for other configurations. The stability of the global system using the state estimation of our observer as feedback is also proved. Simulations of a plausible real task are presented in Sec. IV. Conclusions and future works are reported in Sec. V.

\section{MODEL, CONTROL, AND SENSORS}

As in [7], we consider a multi robot system composed by two aerial vehicles laying on a vertical plane, where the first one is attached to the ground and to the second one by two links, in the way of forming a chain of two elements. A representation of the system is given in Fig. 1. Considering the second robot as an end effector, the system appears similar to a two-link $2-\mathrm{R}$ robot where the aerial vehicles are the actuators. In the following we utilize the notation ${ }_{i}$ with $i=1,2$ in order to refer to the first or the second, respectively. 


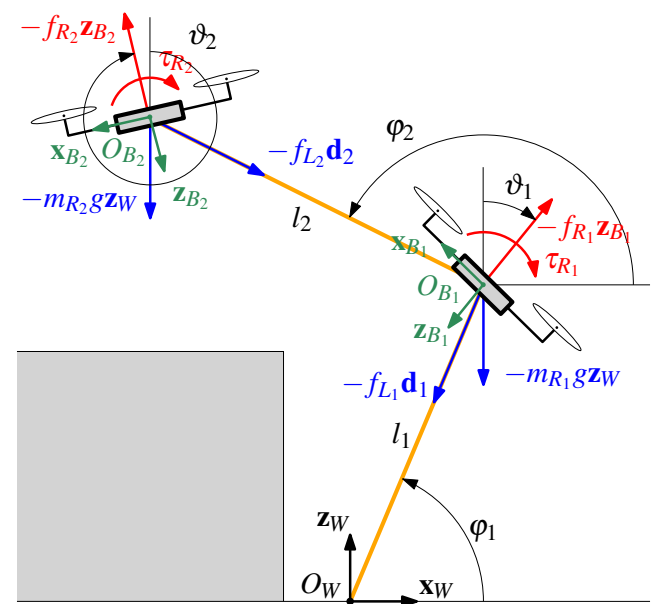

Fig. 1: Representation of the system and its main variables. The system is depicted in a possible scenario where the grey box represents a surface of interest, e.g., for a search and rescue task.

Each robot is modeled as a rigid body with mass $m_{R i} \in \mathbb{R}_{>0}$ and rotational inertia $J_{R i} \in \mathbb{R}_{>0}$, with $i=1,2$.

As in [4]-[7], we assume that the links have negligible mass and rotational inertia with respect the ones of the aerial robots, and also negligible deformations and elasticity. In this condition each link results to have a fixed length $l_{i} \in \mathbb{R}_{>0}$ with $i=1,2$. One end of the first link is connected to the ground and the other to the center of mass (CoM) of the first robot. While, the second link is connected to the CoMs of the two vehicles. The three link-robot and one link-ground connections are made with passive rotational joints in such a way that no rotational constraint holds.

To describe the system configuration, we define the $i$-th elevation, $\varphi_{i} \in \mathbb{R}$, the angle that the $i$-th link forms with the horizon. With $\vartheta_{i}$ we denote the attitude of the $i$-th vehicle. Then, we define $f_{L_{i}} \in \mathbb{R}$ as the stress of the $i$-th link, i.e., the internal force along the link itself. If a link is pulled, i.e., $f_{L_{i}}>0$, the stress is called tension. On the other hand, if a link is compressed, i.e., $f_{L_{i}}<0$, the stress is called compression. The methodology presented here can be applied to any kind of links like, cables, struts or bars, that can resist only to tension, compression, and generic stress, respectively.

Under these assumptions we want to investigate with which different types of sensorial configurations it is possible to observe the system state. For the configurations granting observability we want to derive an observer able to recover the state in any dynamic condition, in order to stably close the loop with the controller in [7] when following any desired trajectory of the Cartesian position of the CoM of the second robot and any desired pair of stresses for the two links.

We define a world frame, $\mathscr{F}_{W}$ with axes $\left\{\mathbf{x}_{W}, \mathbf{y}_{W}, \mathbf{z}_{W}\right\}$, and origin $O_{W}$ on the fixed point on the ground. Then we define two body frames, $\mathscr{F}_{B i}$ rigidly attached to the $i$-th robot $(i=1,2)$. The origin $O_{B_{i}}$ of $\mathscr{F}_{B i}$ is set on the CoM the robot,

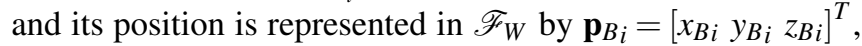
where $y_{B i}=0$. We denote with $\left\{\mathbf{x}_{B i}, \mathbf{y}_{B i}, \mathbf{z}_{B i}\right\}$ the axes of $\mathscr{F}_{B i}$, where $\mathbf{y}_{B i}$ is parallel to $\mathbf{y}_{W}$, and both are perpendicular to the vertical plane $\left\{\mathbf{x}_{W}, \mathbf{y}_{W}\right\}$ where the two robots lie. The system is subject to the thrust forces $-f_{R_{i}} \mathbf{z}_{B i} \in \mathbb{R}^{3}$ and the moments (torques) $\tau_{R i} \mathbf{y}_{B_{i}} \in \mathbb{R}^{3}$, with $i=1,2$. Their intensities, $f_{R_{i}} \in \mathbb{R}$ and $\tau_{R i} \in \mathbb{R}$, with $i=1,2$, constitute the four control inputs of the system.

Given the constraints, the system results completely described by the generalized coordinates $\mathbf{q}=\left(\varphi_{1}, \varphi_{2}, \vartheta_{1}, \vartheta_{2}\right)$.

As done in [7] the dynamic model of the system is obtained applying the Euler-Lagrange method to the kinematic and potential energy, and it results to be

$$
\begin{aligned}
\mathbf{M}(\boldsymbol{\varphi}) \ddot{\boldsymbol{\varphi}} & =-\mathbf{c}(\boldsymbol{\varphi}, \dot{\boldsymbol{\varphi}})+\overline{\mathbf{Q}}_{\boldsymbol{\varphi}}(\boldsymbol{\varphi}, \boldsymbol{\vartheta}) \mathbf{f}_{R} \\
\mathbf{J}_{R} \ddot{\boldsymbol{\vartheta}} & =\boldsymbol{\tau}_{R},
\end{aligned}
$$

where $\boldsymbol{\varphi}=\left[\begin{array}{ll}\varphi_{1} & \varphi_{2}\end{array}\right]^{T}, \boldsymbol{\vartheta}=\left[\begin{array}{ll}\vartheta_{1} & \vartheta_{2}\end{array}\right]^{T}, \mathbf{f}_{R}=\left[\begin{array}{ll}f_{R 1} & f_{R 2}\end{array}\right]^{T}, \boldsymbol{\tau}_{R}=$ $\left[\begin{array}{ll}\tau_{R 1} & \tau_{R 2}\end{array}\right]^{T}, g \approx 9.81$ is the gravitational constant, and the matrices $\mathbf{M}(\boldsymbol{\varphi}), \mathbf{J}_{R}, \mathbf{c}(\boldsymbol{\varphi}, \dot{\boldsymbol{\varphi}})$ and $\overline{\mathbf{Q}}_{\boldsymbol{\varphi}}(\boldsymbol{\varphi}, \boldsymbol{\vartheta})$ are fully described in [7]. In order to control the system as a planar two-links manipulator we exploit the control method presented in [7]. Employing a dynamic feedback linearization it is possible to track any trajectory for the position of the last vehicle defined in the Cartesian space, like for the end effector of a manipulator, and any trajectory for the stress on the links.

For the single link case, in [6] it has been shown that an accelerometer and a gyroscope mounted onboard are sufficient in order to obtain an estimation of the state. For the double link case we are not yet able to derive an observer using only accelerometers and gyroscopes on each vehicle. However, it is also extremely interesting to explore different minimal sensorial configurations in order to retrieve an estimation of the state. In particular, one can try to use two accelerometers (one for each vehicle) together with, instead of two gyroscopes, two additional sensors capable to directly measure the relative or absolute value of the elevation of the links or the attitude of the robots. For example one can use i) two encoders mounted on one end of the links to get the measurements of the elevations [3], or ii) two inclinometers or equivalently some vision techniques to obtain the measurements of the attitudes [8], [9].

In Tab. I all the possible sensor configurations considered in this work are listed with the respective measurements. With reference to the table we use the following convention:

i) Considering an inclinometer rigidly attached to $\mathscr{F}_{B i}$, we call it absolute if it measures the attitude of the $i$-th robot with respect to $\mathscr{F}_{W}, \rho=\vartheta_{i}$; while we call it relative if it measures the attitude of the other vehicle with respect to $\mathscr{F}_{B i}, \rho=\vartheta_{j}-\vartheta_{i}$, where $(i, j) \in\{(1,2),(2,1)\}$.

ii) An encoder rigidly attached to $\mathscr{F}_{W}$ connected to the first link directly measures the absolute elevation angle, $\rho=\varphi_{1}$. While, if it is rigidly attached to $\mathscr{F}_{B i}$ and connected to the $j$-th link it measures the $j$-th elevation angle with respect to $\mathscr{F}_{B i}, \rho=\varphi_{i}+\vartheta_{j}$ with $(i, j) \in$ $\{(1,1),(2,1),(2,2)\}$.

\section{OBSERVER}

In this section, for the case 4 of Tab. I we present a method to transform the original measurements into direct measurements of the configuration $\mathbf{q}$ and we show that this implies the observability of the full state, i.e., $\mathbf{q}$ and $\dot{\mathbf{q}}$. For this case we propose a nonlinear estimator, based on the High Gain Observer (HGO) [10] able to retrieve the state from any dynamic condition. In the end we analyze the applicability of the method to the other configurations of Tab. I. 


\begin{tabular}{|c|c|c|c|c|}
\hline Config. & $2^{\text {nd }}$ Sensor Type & $\begin{array}{l}\text { Mounting } \\
\text { Place }\end{array}$ & $\begin{array}{c}\text { Measures } \\
\rho_{1}, \rho_{2} \\
\end{array}$ & $\begin{array}{l}\text { Applica- } \\
\text { bility }\end{array}$ \\
\hline 1 & $\begin{array}{l}\text { Absolute Inclinom. } \\
\text { Absolute Inclinom. }\end{array}$ & $\begin{array}{l}\mathscr{F}_{B 1} \\
\mathscr{F}_{B 2}\end{array}$ & $\begin{array}{l}\vartheta_{1} \\
\vartheta_{2}\end{array}$ & yes \\
\hline 2 & $\begin{array}{l}\text { Absolute Inclinom. } \\
\text { Relative Inclinom. }\end{array}$ & $\begin{array}{l}\mathscr{F}_{B 1} \\
\mathscr{F}_{B 2}\end{array}$ & $\vartheta_{1}$ & yes \\
\hline 3 & $\begin{array}{l}\text { Encoder } \\
\text { Encoder } \\
\end{array}$ & $\begin{array}{l}\mathscr{F}_{W}-\operatorname{link}_{1} \\
\mathscr{F}_{B 1}-\operatorname{link}_{1}\end{array}$ & $\begin{array}{c}\varphi_{1} \\
\varphi_{1}+\vartheta_{1}\end{array}$ & no \\
\hline 4 & $\begin{array}{l}\text { Encoder } \\
\text { Encoder }\end{array}$ & $\begin{array}{l}\mathscr{F}_{W}-\operatorname{link}_{1} \\
\mathscr{F}_{B 1}-\operatorname{link}_{2}\end{array}$ & $\begin{array}{c}\varphi_{1} \\
\varphi_{2}+\vartheta_{1}\end{array}$ & yes \\
\hline 5 & $\begin{array}{l}\text { Encoder } \\
\text { Encoder }\end{array}$ & $\begin{array}{l}\mathscr{F}_{W}-\operatorname{link}_{1} \\
\mathscr{F}_{B 2}-\operatorname{link}_{2}\end{array}$ & $\begin{array}{c}\varphi_{1} \\
\varphi_{2}+\vartheta_{2}\end{array}$ & no \\
\hline 6 & $\begin{array}{l}\text { Encoder } \\
\text { Encoder }\end{array}$ & $\begin{array}{l}\mathscr{F}_{B 1}-\operatorname{link}_{1} \\
\mathscr{F}_{B 1}-\text { link }_{2}\end{array}$ & $\begin{array}{l}\varphi_{1}+\vartheta_{1} \\
\varphi_{2}+\vartheta_{1}\end{array}$ & no \\
\hline 7 & $\begin{array}{l}\text { Encoder } \\
\text { Encoder }\end{array}$ & $\begin{array}{l}\mathscr{F}_{B 1}-\text { link }_{1} \\
\mathscr{F}_{B 2}-\text { link }_{2}\end{array}$ & $\begin{array}{l}\varphi_{1}+\vartheta_{1} \\
\varphi_{2}+\vartheta_{2}\end{array}$ & no \\
\hline
\end{tabular}

TABLE I: Possible sensors configurations. The $1^{\text {st }}$ sensor type corresponds to an accelerometer mounted on each robot.

\section{A. Output Transformations}

Assume to have an onboard accelerometer for each robot, placed at $O_{B_{i}}$ and attached to $\mathscr{F}_{B i}$. It measures the specific acceleration, i.e., the total acceleration minus the gravitational one expressed in the body fame, $\mathscr{F}_{B i}$, i.e.:

$$
\mathbf{a}_{i}=R_{W}^{B_{i}}\left(\ddot{\mathbf{p}}_{B_{i}}+g \mathbf{z}_{W}\right)=\left[\begin{array}{lll}
a_{i_{x}} & 0 & a_{i_{z}}
\end{array}\right]^{T},
$$

where $R_{W}^{B_{i}} \in \mathbb{R}^{3}$ is the rotation matrix from $\mathscr{F}_{W}$ to $\mathscr{F}_{B i}$, and $\ddot{\mathbf{p}}_{B_{i}}$ is the acceleration of the CoM of the $i$-th vehicle w.r.t. $\mathscr{F}_{W}$. The force balance equations on each CoM read

$$
\begin{aligned}
& m_{R 1} \ddot{\mathbf{p}}_{B_{1}}=-f_{L_{1}} \mathbf{d}_{1}+f_{L_{2}} \mathbf{d}_{2}-f_{R_{1}} \mathbf{z}_{B_{1}}-m_{R 1} g \mathbf{z}_{W} \\
& m_{R 2} \ddot{\mathbf{p}}_{B_{2}}=-f_{L_{2}} \mathbf{d}_{2}-f_{R_{2}} \mathbf{z}_{B_{2}}-m_{R 2} g \mathbf{z}_{W},
\end{aligned}
$$

where $\mathbf{d}_{i}=\left[\cos \varphi_{i} 0 \sin \varphi_{i}\right]^{T} \in \mathbb{R}^{3}$ is parallel to the $i$-th link.

Then we assume to be in the configuration \# 4 of Tab. I, i.e., the system is equipped with two encoders, one is rigidly attached to the ground and connected to the first link and measures its absolute elevation relative to $\mathscr{F}_{W}$, while the second is fixed to $\mathscr{F}_{B 1}$ and connected to the second link, and measures its relative elevation with respect to $\mathscr{F}_{B 1}$, i.e.:

$$
\rho_{1}=\varphi_{1}, \quad \rho_{2}=\varphi_{2}+\vartheta_{1} .
$$

Now, replacing $\ddot{\mathbf{p}}_{B_{2}}$ from (3b) into (2) for $i=2$, we obtain

$$
-m_{R 2} \mathbf{a}_{2}=R_{W}^{B_{i}}\left(f_{L_{2}} \mathbf{d}_{2}+f_{R_{2}} \mathbf{z}_{B_{2}}\right)=f_{L_{2}} R_{W}^{B_{i}} \mathbf{d}_{2}+\left[\begin{array}{lll}
0 & 0 & f_{R 2}
\end{array}\right]^{T},
$$

which allows to define the measurement transformation

$$
\begin{aligned}
{\left[\begin{array}{c}
w_{1}(k) \\
w_{2}(k)
\end{array}\right] } & =\left[\begin{array}{c}
\operatorname{sgn}\left(k-\frac{1}{2}\right) \sqrt{\left(m_{R 2} a_{2_{x}}\right)^{2}+\left(m_{R 2} a_{2_{2}}+f_{R 2}\right)^{2}} \\
\operatorname{atan2} 2\left(\frac{m_{22} a_{2}+f_{R_{2}}}{w_{1}(k)}, \frac{m_{R 2} a_{2 x}}{w_{1}(k)}\right)
\end{array}\right] \\
& =\left[\begin{array}{c}
0 \\
\varphi_{2}+\vartheta_{2}+\frac{\pi}{2}
\end{array}\right] \pm\left[\begin{array}{c}
f_{L_{2}} \\
\frac{\pi}{2}
\end{array}\right],
\end{aligned}
$$

where $k \in\{0,1\}$. Note that (i) there are two solutions for $k=0$ and $k=1$ because $\operatorname{sgn}\left(f_{L_{2}}\right)$ is not retrievable from the measurements; (ii) the transformation is allowed iff $f_{L_{2}} \neq 0$.

At every time instant $t$ there is only one correct pair of measurements, equal to $\left(f_{L_{2}}, \varphi_{2}+\vartheta_{2}\right)$, while the other is wrong and equal to $\left(-f_{L_{2}}, \varphi_{2}+\vartheta_{2}+\pi\right)$. We define $k^{*}$ the unique $k \in\{0,1\}$ such that $\left(w_{1}\left(k^{*}\right), w_{2}\left(k^{*}\right)\right)=\left(f_{L_{2}}, \varphi_{2}+\right.$ $\vartheta_{2}$ ). Then, replacing (3a) into (2) for $i=1$, and after some simple algebra, we can define two additional new measurement transformations:

$$
\begin{aligned}
\bar{a}_{1_{x}} & =m_{R 1} a_{1_{x}}-w_{1}\left(k^{*}\right) \cos \rho_{2} \\
\bar{a}_{1_{z}} & =m_{R 2} a_{2_{z}}-w_{1}\left(k^{*}\right) \sin \rho_{2}+f_{R_{2}} \\
{\left[\begin{array}{c}
w_{3}\left(k^{*}, j\right) \\
w_{4}\left(k^{*}, j\right)
\end{array}\right] } & =\left[\begin{array}{c}
\operatorname{sgn}\left(j-\frac{1}{2}\right) \sqrt{\bar{a}_{1_{x}}^{2}+\bar{a}_{1}^{2}} \\
\operatorname{atan} 2\left(\frac{\bar{a}_{1_{z}}}{w_{3}\left(k^{*}, j\right)}, \frac{\bar{a}_{1_{x}}}{w_{3}\left(k^{*}, j\right)}\right)
\end{array}\right] \\
& =\left[\begin{array}{c}
0 \\
\varphi_{1}+\vartheta_{1}+\frac{\pi}{2}
\end{array}\right] \pm\left[\begin{array}{c}
f_{L_{1}} \\
-\frac{\pi}{2}
\end{array}\right],
\end{aligned}
$$

where $j \in\{0,1\}$. As in (5), the previous transformation is not possible when $f_{L_{1}}=0$. A practical solution for the instantaneous zero stress case is provided in Sec. III-E.1

Since the sign of $f_{L_{1}}$ is not retrievable from the measurements, we obtain two solutions parametrized by $j$, i.e., $\left(w_{3}\left(k^{*}, j\right), w_{4}\left(k^{*}, j\right)\right)$. At every time instant $t$ there is only one correct pair of measurements equal to $\left(f_{L_{1}}, \varphi_{1}+\vartheta_{1}\right)$, while the other is wrong and equal to $\left(-f_{L_{1}}, \varphi_{1}+\vartheta_{1}+\pi\right)$. Actually, recalling that also $k \in$ $\{0,1\}$, we obtain four groups of different measurements, i.e., $\left(w_{1}(k), w_{2}(k), w_{3}(k, j), w_{4}(k, j)\right)$ with $k, j \in\{0,1\}$. We know that at each time $t$ there is only one couple $k^{*}, j^{*} \in\{0,1\}$ such that the corresponding measurements are correct, i.e., $\left(w_{1}\left(k^{*}\right), w_{2}\left(k^{*}\right), w_{3}\left(k^{*}, j^{*}\right), w_{4}\left(k^{*}, j^{*}\right)\right)=$ $\left(f_{L_{2}}, \varphi_{2}+\vartheta_{2}, f_{L_{1}}, \varphi_{1}+\vartheta_{1}\right)$, while all the others are wrong.

Finally, exploiting the readings of the encoders, we can define the last output transformation

$$
\begin{aligned}
\eta_{1} & =\rho_{1} \\
\eta_{2}(k, j) & =\rho_{1}+\rho_{2}-w_{4}(k, j) \\
\eta_{3}(k, j) & =w_{4}(k, j)-\rho_{1} \\
\eta_{4}(k, j) & =w_{2}(k)+w_{4}(k, j)-\rho_{1}-\rho_{2} .
\end{aligned}
$$

The transformation method is represented in Fig. 2a. From (9) one can notice that for the pair $\left(k^{*}, j^{*}\right)$ defined before, we obtain a direct measure of the generalized coordinates, i.e. $\boldsymbol{\eta}\left(k^{*}, j^{*}\right)=\left[\eta_{1} \eta_{2}\left(k^{*}, j^{*}\right) \eta_{3}\left(k^{*}, j^{*}\right) \eta_{4}\left(k^{*}, j^{*}\right)\right]^{T}=$ $\left[\begin{array}{lll}\boldsymbol{\eta}_{1}^{T}\left(k^{*}, j^{*}\right) & \boldsymbol{\eta}_{2}^{T}\left(k^{*}, j^{*}\right)\end{array}\right]^{T}=\left[\begin{array}{llll}\varphi_{1} & \varphi_{2} & \vartheta_{1} & \vartheta_{2}\end{array}\right]^{T}$. While, for the pairs $(k, j) \neq\left(k^{*}, j^{*}\right), \boldsymbol{\eta}(k, j)$ is a wrong measurement of the configuration. From a single set of measures it is not possible to discriminate which is the correct pair $\left(k^{*}, j^{*}\right)$ corresponding to the correct $\boldsymbol{\eta}$, nevertheless, in Sec. III-D we show a discriminating method exploiting the dynamics.

For the purpose of proving the observability of the system and for designing the observer we consider $\boldsymbol{\eta}=\boldsymbol{\eta}\left(k^{*}, j^{*}\right)$.

\section{B. Observability}

In order to study the observability of the system and to design an observer of the state, we first rewrite the system in a state space form by defining $\mathbf{x}=$ $\left[\begin{array}{llllllll}x_{1} & x_{2} & x_{3} & x_{4} & x_{5} & x_{6} & x_{7} & x_{8}\end{array}\right]^{T}=\left[\begin{array}{llllllll}\varphi_{1} & \dot{\varphi}_{1} & \varphi_{2} & \dot{\varphi}_{2} & \vartheta_{1} & \dot{\vartheta}_{1} & \vartheta_{2} & \dot{\vartheta}_{2}\end{array}\right]^{T} \in \mathbb{R}^{8}$ and $\mathbf{u}=\left[\begin{array}{llll}f_{R_{1}} & f_{R_{2}} & \tau_{R 1} & \tau_{R 2}\end{array}\right]^{T}=\left[\begin{array}{ll}\mathbf{f}_{R}{ }^{T} & \boldsymbol{\tau}_{R}^{T}\end{array}\right]^{T}=\left[\begin{array}{ll}\mathbf{u}_{1}^{T} & \mathbf{u}_{2}^{T}\end{array}\right]^{T} \in \mathbb{R}^{4}$ as the state and input vectors of the system, respectively. We can then rewrite (1) and the measurements function (9) in the state-space form as

$$
\begin{aligned}
& \dot{\mathbf{x}}=\mathbf{A x}+\mathbf{B}\left[\begin{array}{c}
\boldsymbol{\sigma}\left(\mathbf{x}, \mathbf{u}_{1}\right) \\
\mathbf{J}_{R}^{-1} \mathbf{u}_{2}
\end{array}\right] \\
& \boldsymbol{\eta}=\mathbf{C x}
\end{aligned}
$$


where $\mathbf{A}=\operatorname{diag}^{1}\left(\mathbf{A}_{1}, \mathbf{A}_{2}, \mathbf{A}_{3}, \mathbf{A}_{4}\right), \mathbf{B}=\operatorname{diag}\left(\mathbf{B}_{1}, \mathbf{B}_{2}, \mathbf{B}_{3}, \mathbf{B}_{4}\right)$, $\mathbf{C}=\operatorname{diag}\left(\mathbf{C}_{1}, \mathbf{C}_{2}, \mathbf{C}_{3}, \mathbf{C}_{4}\right)$

$$
\begin{array}{r}
\mathbf{A}_{i}=\left[\begin{array}{ll}
0 & 1 \\
0 & 0
\end{array}\right], \quad \mathbf{B}_{i}=\left[\begin{array}{l}
0 \\
1
\end{array}\right], \quad \mathbf{C}_{i}=\left[\begin{array}{ll}
1 & 0
\end{array}\right] \quad \forall i=1, \ldots, 4 \\
\boldsymbol{\sigma}\left(\mathbf{x}, \mathbf{u}_{1}\right)=-\mathbf{M}(\mathbf{x})^{-1} \mathbf{c}(\mathbf{x})+\mathbf{M}(\mathbf{x})^{-1} \overline{\mathbf{Q}}_{\boldsymbol{\varphi}}(\mathbf{x}) \mathbf{u}_{1},
\end{array}
$$

since $\mathbf{M}(\mathbf{x})$ is always invertible. Writing (10) as $\dot{\mathbf{x}}=$ $\mathbf{f}(\mathbf{x}, \mathbf{u})$, and $\boldsymbol{\eta}=\mathbf{h}(\mathbf{x})$. the system results observable if the non linear observability matrix $\mathscr{O}(\mathbf{x}, \mathbf{u})=$ $\left[\frac{\partial \mathbf{h}(\mathbf{x})}{\partial \mathbf{x}}, \frac{\partial \dot{\mathbf{h}}(\mathbf{x})}{\partial \mathbf{x}}, \ldots, \frac{\partial \mathbf{h}^{(7)}(\mathbf{x})}{\partial \mathbf{x}}\right]^{T} \in \mathbb{R}^{4 \cdot 8 \times 8}$ is full rank [11]. We can notice that

$$
\mathscr{O}(\mathbf{x}, \mathbf{u})_{1}=\left[\frac{\partial \mathbf{h}(\mathbf{x})}{\partial \mathbf{x}}, \frac{\partial \dot{\mathbf{h}}(\mathbf{x})}{\partial \mathbf{x}}\right]^{T}=\left[\mathbf{C}^{T}(\mathbf{C A})^{T}\right]^{T} .
$$

Changing the order of the rows we obtain $\mathscr{O}(\mathbf{x}, \mathbf{u})_{1}^{\prime}=\mathbf{I}_{8}$, that is full rank for every $\mathbf{x} \in \mathbb{R}^{8}$ and $\mathbf{u} \in \mathbb{R}^{4}$. This implies that also $\mathscr{O}(\mathbf{x}, \mathbf{u})$ is always full rank, i.e.,

Proposition 1. Consider the system described by (1) with two onboard accelerometers, mounted on each vehicles, and two encoders, one attached to the ground and connected to the first link, and one mounted to the first vehicle and connected to the second link. Then, the system is observable except for the zero stress cases, i.e., $f_{L_{1}}=0$ or $f_{L_{2}}=0$.

Although we proved Prop. 1 only for the fourth case of Tab. I, actually, the result shows a more general sufficient observability condition. Indeed, independently from the available sensors, whenever there are some output transformations that translate the original measurements into direct measures of $\mathbf{q}$, then the system is observable, i.e.,

Proposition 2. Consider the system described by (10a) and a set of measurements $\mathbf{w}=\mathbf{h}(\mathbf{x}, \mathbf{u}) \in \mathbb{R}^{p}$, where $p \in \mathbb{R}_{\geq 1}$. Define $\mathscr{X}$ the state space and $\mathscr{U}$ the control inputs space. If there exists a subspace $\mathscr{D} \subseteq \mathscr{X} \times \mathscr{U}$ and an measurement transformation function $\Gamma: \overline{\mathbb{R}}^{p} \rightarrow \mathbb{R}^{4}$ valid in $\mathscr{D}$, such that $\left[\begin{array}{llll}\varphi_{1} & \varphi_{2} & \vartheta_{1} & \vartheta_{2}\end{array}\right]^{T}=\Gamma(\mathbf{w})$, then the system is observable for every $\mathbf{x}$ and $\mathbf{u}$ in $\mathscr{D}$, and can be written in the form of (10).

\section{High Gain Observer}

For the sets of measurements that fulfill the condition of Prop. 2, and in particular for the case 4 of Tab. I we show in this section the design of an observer based on HGO [10].

Considering the system (10) we define $\zeta=\left[\begin{array}{ll}\zeta_{1}^{T} & \zeta_{2}^{T}\end{array}\right]^{T}=$ $\left[\begin{array}{llll}\zeta_{1} & \zeta_{2} & \zeta_{3} & \zeta_{4}\end{array}\right]^{T}=\left[\begin{array}{llll}x_{1} & x_{2} & x_{3} & x_{4}\end{array}\right]^{T}$ and $\mathbf{z}=\left[\mathbf{z}_{1}^{T}, \mathbf{z}_{2}^{T}\right]^{T}=$ $\left[\begin{array}{llll}z_{1} & z_{2} & z_{3} & z_{4}\end{array}\right]^{T}=\left[\begin{array}{llll}x_{5} & x_{6} & x_{7} & x_{8}\end{array}\right]^{T}$. The system (10) can be then written as

$$
\left\{\begin{array} { l } 
{ \dot { \zeta } = \mathbf { A } _ { \boldsymbol { \zeta } } \boldsymbol { \zeta } + \mathbf { B } _ { \boldsymbol { \zeta } } \boldsymbol { \sigma } ( \boldsymbol { \zeta } , \boldsymbol { \eta } _ { 2 } , \mathbf { u } _ { 1 } ) } \\
{ \boldsymbol { \eta } _ { 1 } = \mathbf { C } _ { \boldsymbol { \zeta } } \boldsymbol { \zeta } }
\end{array} \quad \left\{\begin{array}{l}
\dot{\mathbf{z}}=\mathbf{A}_{\mathbf{z}} \mathbf{z}+\mathbf{B}_{\mathbf{z}} \mathbf{u}_{2} \\
\boldsymbol{\eta}_{2}=\mathbf{C}_{\mathbf{z}} \mathbf{z}
\end{array}\right.\right.
$$

where $\mathbf{A}_{\boldsymbol{\zeta}}=\operatorname{diag}\left(\mathbf{A}_{1}, \mathbf{A}_{2}\right), \quad \mathbf{B}_{\boldsymbol{\zeta}}=\operatorname{diag}\left(\mathbf{B}_{1}, \mathbf{B}_{2}\right), \quad \mathbf{C}_{\boldsymbol{\zeta}}=$ $\operatorname{diag}\left(\mathbf{C}_{1}, \mathbf{C}_{2}\right), \quad \mathbf{A}_{\mathbf{z}}=\operatorname{diag}\left(\mathbf{A}_{3}, \mathbf{A}_{4}\right), \quad \mathbf{B}_{\mathbf{z}}=\operatorname{diag}\left(\mathbf{B}_{3}, \mathbf{B}_{4}\right) \mathbf{J}_{R}^{-1}$, $\mathbf{C}_{\mathbf{z}}=\operatorname{diag}\left(\mathbf{C}_{3}, \mathbf{C}_{4}\right)$. Having replaced $\vartheta_{1}$ and $\vartheta_{2}$ with their measures $\boldsymbol{\eta}_{2}$ in the dynamics of $\boldsymbol{\zeta}$, the two systems become completely independent, moreover, the second one is linear, therefore we can design for it a classical Luenberger observer

$$
\dot{\hat{\mathbf{z}}}=\mathbf{A}_{\mathbf{z}} \hat{\mathbf{z}}+\mathbf{B}_{\mathbf{z}} \mathbf{u}_{2}+\mathbf{H}_{\mathbf{z}}\left(\boldsymbol{\eta}_{2}-\mathbf{C}_{\mathbf{z}} \hat{\mathbf{z}}\right)
$$

\footnotetext{
${ }^{1} \operatorname{diag}\left(\mathbf{X}_{1}, \ldots, \mathbf{X}_{n}\right)$ is a block matrix having on the main block diagonal the matrices $\mathbf{X}_{i}$, whereas the off-diagonal blocks are zero matrices.
}

where $\mathbf{H}_{\mathbf{z}}=\operatorname{diag}\left(\mathbf{H}_{\mathbf{z}_{1}}, \mathbf{H}_{\mathbf{z}_{1}}\right)$ and $\mathbf{H}_{\mathbf{z}_{i}}=\left[\beta_{1}^{i} \beta_{2}^{i}\right]^{T}$, whose elements, $\beta_{j}^{i} \in \mathbb{R}_{>0}$ can be set to place the poles of the error dynamics, $\mathbf{e}_{\mathbf{z}_{i}}=\mathbf{z}_{i}-\hat{\mathbf{z}}_{i}$. Instead, for the first system, thanks to its particular triangular form, it is possible to use the following $\mathrm{HGO}$

$$
\dot{\hat{\zeta}}=\mathbf{A}_{\zeta} \hat{\zeta}+\mathbf{B}_{\zeta} \boldsymbol{\sigma}\left(\hat{\boldsymbol{\zeta}}, \boldsymbol{\eta}_{2}, \mathbf{u}_{1}\right)+\mathbf{H}_{\boldsymbol{\zeta}}\left(\boldsymbol{\eta}_{1}-\mathbf{C}_{\boldsymbol{\zeta}} \hat{\boldsymbol{\zeta}}\right)
$$

where $\mathbf{H}_{\zeta}=\operatorname{diag}\left(\mathbf{H}_{\zeta_{1}}, \mathbf{H}_{\zeta_{1}}\right)$ and $\mathbf{H}_{\zeta_{i}}=\left[\frac{\alpha_{1}^{i}}{\varepsilon} \frac{\alpha_{2}^{i}}{\varepsilon^{2}}\right]^{T}$, with $\varepsilon \in$ $\mathbb{R}_{>0}$, and the gains $\alpha_{j}^{i} \in \mathbb{R}_{>0}$ are set such that the roots of $s^{2}+\alpha_{1}^{i} s+\alpha_{2}^{i}$ have negative real part. The gains $\left(\alpha_{1}^{i}, \alpha_{2}^{i}\right)$ influence the convergence rate of the estimation of the $i$-th elevation angle and its derivative, i.e., $\varphi_{i}$ and $\dot{\varphi}_{i}$. A schematic representation of the observer is given in Fig. $2 b$.

\section{Disambiguation of $\eta$}

The output transformations described in Sec. III-A generates four different set of measurements, $\boldsymbol{\eta}(k, j)$ with $k, j \in$ $\{0,1\}$, of which only one is correct.

As represented in Fig. 2a, for each $k, j \in\{0,1\}$, we implement an observer of the state, $\Sigma_{k j}$, using (12) and (13), based on the measurements $\boldsymbol{\eta}(k, j)$. Therefore we obtain four estimates of the state, one for each measurement pair, $\hat{\mathbf{x}}_{0,0}$, $\hat{\mathbf{x}}_{0,1}, \hat{\mathbf{x}}_{1,0}, \hat{\mathbf{x}}_{1,1}$, and the correct one has to be recognized.

Define $\hat{\mathbf{w}}=\left[\begin{array}{llll}\hat{\mathbf{a}}_{1}^{T} & \hat{\mathbf{a}}_{2}^{T} & \hat{\rho}_{1} & \hat{\rho}_{2}\end{array}\right]^{T}$ as the vector that contains the measurements computed with the estimated state, i.e.,

$$
\hat{\rho}_{1}=\hat{x}_{1}, \quad \hat{\rho}_{2}=\hat{x}_{3}+\hat{x}_{5}, \quad \hat{\mathbf{a}}_{i}=\hat{R}_{W}^{B_{i}}\left(\hat{\ddot{\mathbf{p}}}_{B_{i}}-g \mathbf{z}_{W}\right),
$$

where $\hat{R}_{W}^{B_{i}}=R_{W}^{B_{i}}(\hat{\mathbf{x}})$, and $\hat{\mathbf{p}}_{B_{i}}$ is calculated considering the system model (i.e., no numerical differentiation is needed)

$$
\begin{aligned}
& \hat{\hat{\mathbf{p}}}_{B_{1}}=-l_{1} \mathbf{d}_{1}\left(\hat{x}_{1}\right) \hat{x}_{2}^{2}+l_{1} \mathbf{d}_{1}^{\perp}\left(\hat{x}_{1}\right) \hat{\dot{x}}_{2} \\
& \hat{\ddot{\mathbf{p}}}_{B_{2}}=\hat{\ddot{\mathbf{p}}}_{B_{1}}-l_{2} \mathbf{d}_{2}\left(\hat{x}_{3}\right) \hat{x}_{4}^{2}+l_{2} \mathbf{d}_{2}^{\perp}\left(\hat{x}_{3}\right) \hat{\dot{x}}_{4} .
\end{aligned}
$$

In the previous equations $\hat{\dot{x}}_{2}$ and $\hat{\dot{x}}_{4}$ are the estimation of the angular acceleration of the elevations calculated replacing the estimated state into (11), i.e., $\left[\begin{array}{ll}\hat{\dot{x}}_{2} & \hat{\dot{x}}_{4}\end{array}\right]^{T}=\boldsymbol{\sigma}\left(\hat{\mathbf{x}}, \mathbf{u}_{1}\right)$ (no numerical differentiation needed in this case either).

In order to choose the correct estimation among the four, we propose a method based on the minimal prediction error. For each observer we compute a prediction error $\tilde{e}_{k, j}$ smoothed with an exponential discount factor:

$$
\dot{\tilde{e}}_{k, j}=\lambda\left(\left\|\mathbf{w}-\hat{\mathbf{w}}_{k, j}\right\|-\tilde{e}_{k, j}\right)
$$

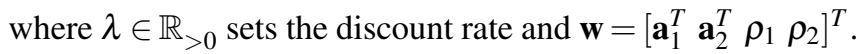
Then the estimation of the observer with minimum prediction error is chosen, i.e., $\hat{\mathbf{x}}=\hat{\mathbf{x}}_{k^{*}, j^{*}}$ s.t. $\left\{k^{*}, j^{*}\right\}=$ $\arg \min _{k, j \in\{0,1\}}\left(\tilde{e}_{k, j}\right)$. Fig. 2 shows the estimator structure.

\section{E. Discussion on the Proposed Method}

1) Zero Stress Case: As we previously noticed, if the one stresses is zero then $w_{2}$ or $w_{4}$ cannot be determined. In the very special case that the stress has to be constantly equal to zero, the proposed observer is not applicable. Nevertheless, if the desired stress is passing through zero for a sufficiently short (ideally zero) time interval, then the proposed observer 


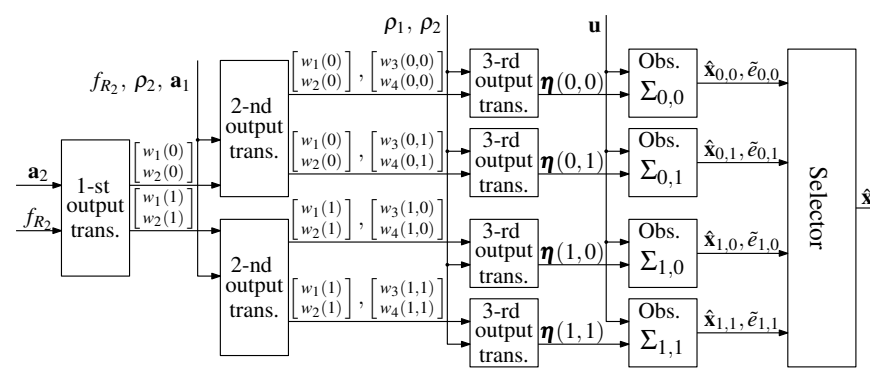

(a) Global Observer.

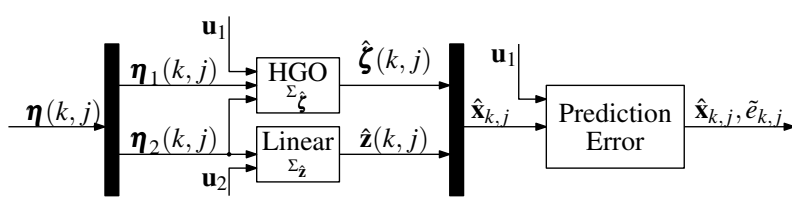

(b) Observer $\Sigma_{k, j}$.

Fig. 2: Graphic representation of the observer.

can be still used in practice by updating the filter without the correction term in that time instants, i.e., imposing

$$
\begin{aligned}
& \dot{\hat{\mathbf{z}}}=\mathbf{A}_{\mathbf{z}} \hat{\mathbf{z}}+\mathbf{B}_{\mathbf{z}} \mathbf{u}_{2} \\
& \dot{\hat{\boldsymbol{\zeta}}}=\mathbf{A}_{\boldsymbol{\zeta}} \hat{\boldsymbol{\zeta}}+\mathbf{B}_{\boldsymbol{\zeta}} \boldsymbol{\sigma}\left(\hat{\boldsymbol{\zeta}}, \hat{\mathbf{z}}, \mathbf{u}_{1}\right) \quad \text { if } w_{1}=0 \text { or } w_{3}=0 .
\end{aligned}
$$

In this way the error dynamics becomes non strictly stable for a short moment since the observation is done in 'open loop' only using the model dynamics. However, the dynamics returns asymptotically stable as soon as the stress becomes non-zero again, as it is shown in the simulations of Sec. IV.

2) Applicability: The transformation method showed for the case 4 in Tab. I can be applied also to other sets of sensors. Last column of Tab. I specifies for which configurations the method is able to transform the original measurements into direct measures of the system configuration.

Due to space limitation, we do not report here the detailed output transformations for every case. However, for cases 1 and 2, they are very similar to those derived for case 4 .

For the remaining configurations it is not possible to apply the proposed method. In particular for the cases $3,5,7$ we cannot compute the transformation (8). While, for the case 6 , the problem lies in the last transformation (9).

\section{F. Closed Loop Stability}

For the control law described by in [7], the knowledge of the state is sufficient in order to close the loop. Thus we can use as feedback the state estimation provided by the proposed observer. Being the system non-linear one cannot apply a separation principle like in the linear case. However it can be shown that for the kind of systems in exam, i.e., triangular block system for which there is a direct measure of the first state of each block, a strong property holds [10]. In fact, since the closed loop system by state feedback is exponentially stable for every state except the zero thrust case, then, there exist a $\varepsilon^{*}$ such that, for every $0<\varepsilon \leq \varepsilon^{*}$ in (13), the closed loop system with the observer is exponentially stable, except for the zero thrust and zero stress case [10].

\section{NUMERICAL SIMULATIONS}

We tested the closed loop system (observer + controller) in simulation using two aerial robots with $m_{R i}=1[\mathrm{~kg}]$ and $J_{R i}=0.15\left[\mathrm{~kg} \mathrm{~m}^{2}\right]$, and two links with $l_{i}=2[\mathrm{~m}](i=1,2)$.

In order to obtain a reasonable fast tracking of the desired trajectories we set the gains such as the error dynamics relative to $\varphi_{1}, \varphi_{2}$ and $f_{L_{1}}, f_{L_{2}}$ has poles in $(-3,-6,-9,-12)$ and $(-5,-10)$, respectively. Regarding the convergence of the state estimation, we set $\varepsilon=0.1$ and the gains $\left(\alpha_{1}^{i}, \alpha_{2}^{i}\right)$ such as the roots of $s^{2}+\alpha_{1}^{i} s+\alpha_{2}^{i}$ are $(-2,-3)$. Then $\mathbf{H}_{\mathbf{z}_{i}}$ is set such as the error dynamics of the estimation of $\vartheta_{1}$, $\vartheta_{2}$ has poles in $(-15,-25)$. Finally, the discount rate of the prediction error dynamics is set to $\lambda=20$. These values of the gains, replicated identically for each of the four observers, guarantee the stability of the closed-loop system.

To show the ability of the proposed observer to exponentially converge to the real state, we initialize it with an error of $5^{\circ}$ relatively to the elevation and pitch angle. We propose two different simulations: the first, whose results are plotted in Fig. 3, shows the performances of the observer in the particular case of inversion of the stress. In the second simulation, reported in Fig. 4, we replicate a plausible real scenario where the system is controlled as a two-link robot.

To better represent the behavior of the system, Fig. 3c and Fig. 4c show a stroboscopic evolution where the flow of time is given by the color. To graphically represent the stress variation, the link is drawn as a dashed line with a thinner width when the tension is higher, and as a solid line with a wider width when the compression is higher.

From Fig. 3 and Fig. 4 one can notice that the estimation of the state converges to the real one in less than one second, in any dynamic condition. Moreover, for the first simulation, the prediction error does not increase even when the desired stress passes through zero. Although during the transient of the estimation the controller shows a non zero tracking error, actually, as soon as the estimation error goes to zero, the outputs follow the desired trajectory with high fidelity during the remaining time of the simulation. An animation of the simulations is also available in the attached video.

\section{CONCLUSIONS}

For the problem of estimating the state of the planar aerial chain composed by two underactuated flying robots, we proposed a method to transform the original sensors readings to direct measures of the system configuration. For the sensorial configurations in which the method is applicable we designed a nonlinear observer whereby we feedback the controller presented in [7], closing the control loop. Thus, it is possible to control the system as a planar manipulator (but controlling also the internal stress) without using dedicated sensors for each state and output.

Some future works will be to derive a state observer using only inertial sensors, solve of the problem for the $3 \mathrm{D}$ case and extend it to a distributed control method.

\section{REFERENCES}

[1] F. Muttin, "Umbilical deployment modeling for tethered UAV detecting oil pollution from ship," Applied Ocean Research, vol. 33, no. 4, pp. 332-343, 2011. 

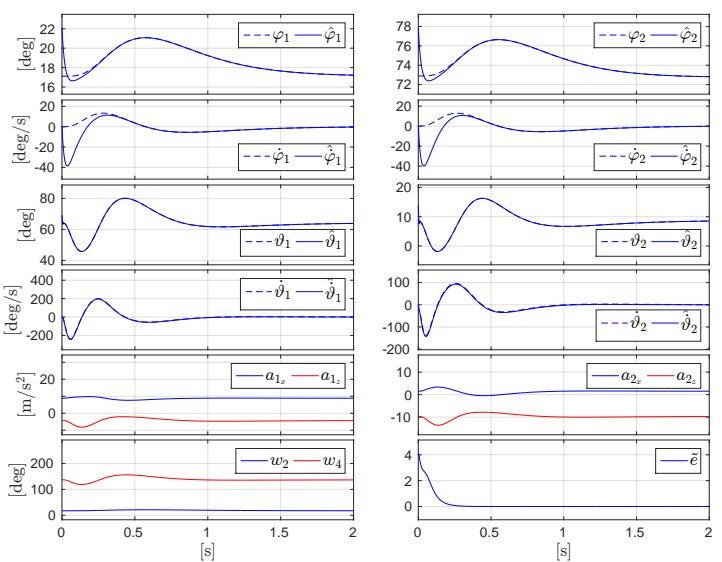

(a) Observer Results: the plot shows only the first 2 seconds of simulation. After this time the estimation follows the state with high fidelity thus the rest of the simulation is not displayed.
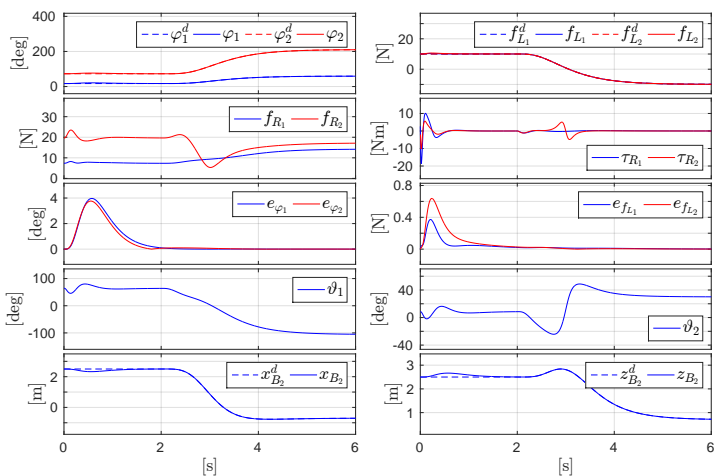

(b) Controller Results.

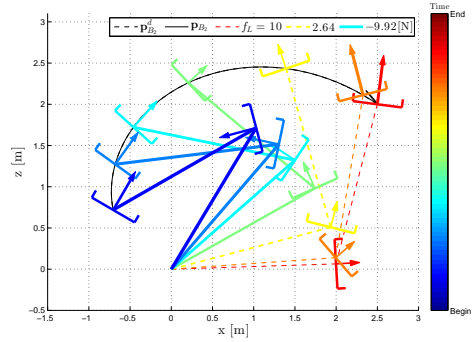

(c) Trajectory visualization from time Begin $=0[\mathrm{~s}]$ to End $=6[\mathrm{~s}]$. The thrust arrow is drawn thicker and longer when the thrust intensity is higher.

Fig. 3: Point to point simulation. The trajectory of the end effector is trajectory of class $C^{3}$ from the initial position $\mathbf{p}_{B_{2}}\left(\begin{array}{l}0 \\ \text { to }\end{array}=\left[\begin{array}{lll}2.5 & 0 & 2\end{array}\right]^{T}\right.$ to the final $\mathbf{p}_{B_{2}}\left(t_{f}\right)=\left[\begin{array}{lll}-0.7 & 0 & 0.7\end{array}\right]^{T}$. While the desired stress is a trajectory of class $C^{1}$ from the initial tension of $10[\mathrm{~N}]$ to the final compression of $-10[\mathrm{~N}]$.

[2] O. Burdakov, P. Doherty, K. Holmberg, J. Kvarnstrom, and P.-R Olsson, "Positioning unmanned aerial vehicles as communication relays for surveillance tasks," in 2009 Robotics: Science and Systems, Seattle, USA, June 2009.

[3] L. Sandino, D. Santamaria, M. Bejar, A. Viguria, K. Kondak, and A. Ollero, "Tether-guided landing of unmanned helicopters without GPS sensors," in 2014 IEEE Int. Conf. on Robotics and Automation, Hong Kong, China, May 2014, pp. 3096-3101

[4] S. Lupashin and R. D'Andrea, "Stabilization of a flying vehicle on a taut tether using inertial sensing," in 2013 IEEE/RSJ Int. Conf. on Intelligent Robots and Systems, Tokyo, Japan, Nov 2013, pp. 24322438.

[5] M. M. Nicotra, R. Naldi, and E. Garone, "Taut cable control of a tethered UAV," in 19th IFAC World Congress, Cape Town, South Africa, Aug. 2014, pp. 3190-3195.
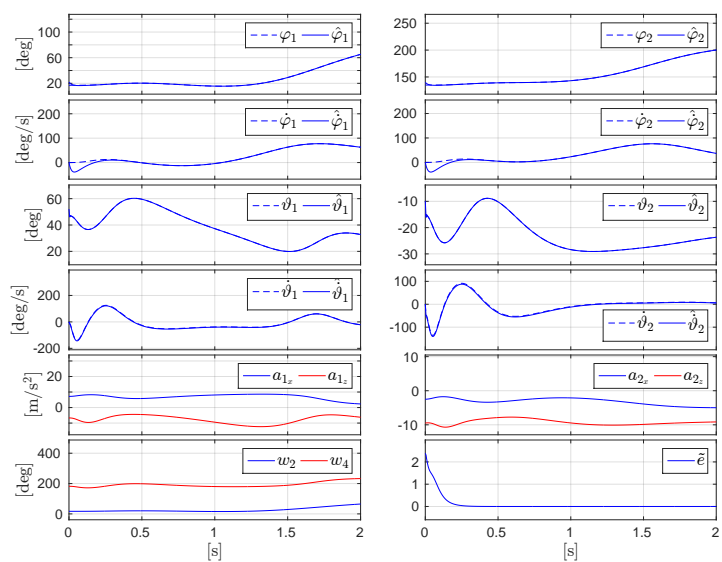

(a) Observer Results: the plot shows only the first 2 seconds of simulation for the same reason of Fig. 4a.
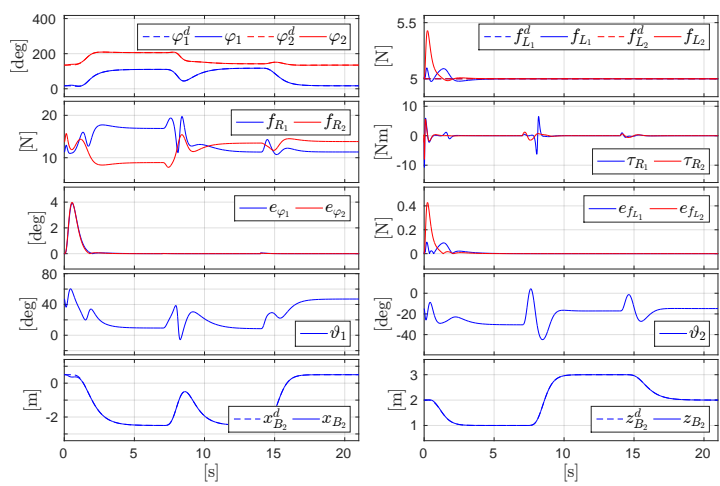

(b) Controller Results.

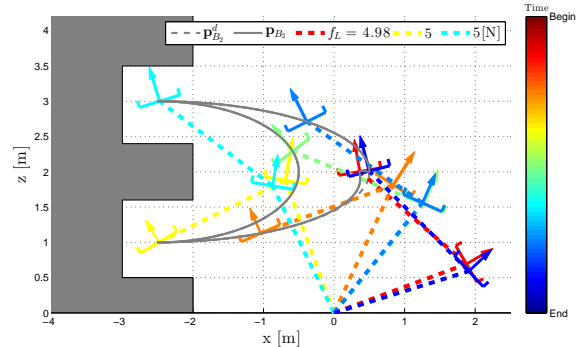

(c) Trajectory visualization from time Begin $=0[\mathrm{~s}]$ to End $=21[\mathrm{~s}]$.

Fig. 4: Example of a search and rescue task simulation. The desired trajectory of the end effector is planned in the Cartesian space as a sequence of three arcs of ellipse in order to enter, stop on each room and then return to the initial position. By inverse kinematics the desired trajectories of the two elevations are derived. In the meanwhile a constant tension of $5[\mathrm{~N}]$ is required on the two links for the hole duration of the task.

[6] M. Tognon and A. Franchi, "Nonlinear observer-based tracking contro of link stress and elevation for a tethered aerial robot using inertialonly measurements," in 2015 IEEE Int. Conf. on Robotics and Automation, Seattle, WA, May 2015, pp. 3994-3999.

[7] — "Control of motion and internal stresses for a chain of two underactuated aerial robots," in 14th European Control Conference, Linz, Austria, Jul. 2015, pp. 1614-1619.

[8] U. Mescheder and S. Majer, "Micromechanical inclinometer," Sensors and Actuators A: Physical, vol. 60, no. 1, pp. 134-138, 1997.

[9] C. Demonceaux, P. Vasseur, and C. Pégard, "Omnidirectional vision on UAV for attitude computation," in 2006 IEEE Int. Conf. on Robotics and Automation, Orlando, FL, May 2006, pp. 2842-2847.

[10] H. K. Khalil, Nonlinear Systems, 3rd ed. Prentice Hall, 2001.

[11] R. Marino and P. Tomei, Nonlinear Control Design: Geometric, Adaptive and Robust. Prentice Hall, 1996. 